SPIN-07/41

ITP-UU-07/55

\title{
Comments on gluon scattering amplitudes via AdS/CFT
}

\author{
Luis F. Alday ${ }^{a}$ and Juan Maldacena ${ }^{b}$ \\ ${ }^{a}$ Institute for Theoretical Physics and Spinoza Institute \\ Utrecht University, 3508 TD Utrecht, The Netherlands \\ ${ }^{b}$ School of Natural Sciences, Institute for Advanced Study \\ Princeton, NJ 08540, USA
}

\begin{abstract}
In this article we consider $n$ gluon color ordered, planar amplitudes in $\mathcal{N}=4$ super Yang Mills at strong 't Hooft coupling. These amplitudes are approximated by classical surfaces in $A d S_{5}$ space. We compute the value of the amplitude for a particular kinematic configuration for a large number of gluons and find that the result disagrees with a recent guess for the exact value of the amplitude. Our results are still compatible with a possible relation between amplitudes and Wilson loops.

In addition, we also give a prescription for computing processes involving local operators and asymptotic states with a fixed number of gluons. As a byproduct, we also obtain a string theory prescription for computing the dual of the ordinary Wilson loop, $\operatorname{Tr} P e^{i \oint A}$, with no couplings to the scalars. We also evaluate the quark-antiquark potential at two loops.
\end{abstract}




\section{Introduction}

In this paper we investigate some properties of planar gluon scattering amplitudes in $\mathcal{N}=4$ super Yang Mills at strong coupling. We use the prescription in [1] (see also [2, 3]) for computing $n$ gluon scattering amplitudes at strong coupling. The computation of the amplitude reduces to a geometric problem. One has to find a surface in $A d S_{5}$ which ends on the boundary on a sequence of light-line lines specified by the momenta of the gluons $\left\{k_{1}, k_{2}, \cdots, k_{n}\right\}$. In [1], we computed the explicit form of the amplitude for the scattering of four gluons, which matched the conjectured form in [4]. At strong coupling the problem has a "dual" conformal symmetry which is enough for determining the four and five gluon scattering amplitudes [1,5]. This symmetry is also present in weak coupling computations [6, 8,5, 5], but its origin and full scope remain mysterious 1 .

One of the goals of this article is to perform a computation which is not determined by the "dual space" conformal symmetry. This only starts happening when one considers $n \geq 6$ gluons [5], where one can start writing non-vanishing conformal invariant cross ratios of the momenta. Doing explicit computations in this case seems rather difficult, because it is hard to find the minimal area surfaces. However, here we will consider the case of $n \rightarrow \infty$ where the problem simplifies. The reason is that we can consider a configuration of lightlike segments which approximates a spacelike line. For some spacelike configurations we can find the minimal surfaces directly, as in [10, [1]. We use this result to test the BDS conjecture [4] which, as shown in [7, 12], amounts to exponentiating the one loop result for a Wilson loop expectation value, up to an overall coefficient in the exponent which is given by the cusp anomalous dimension. Using the relation between the weak and strong coupling forms of the quark-anti-quark potential, one can check that the BDS conjecture is not correct at strong coupling and for a large number of gluons. Recently, a separate conjecture has been discussed [7,12,5], which states that the finite part of MHV amplitudes is the same as the finite part of Wilson loop expectation values. Our results do not offer new arguments for or against this relationship.

As a byproduct of the approximation of spacelike Wilson loop by a zig-zag sequence of light-like ones we get a prescription for the strong coupling dual of the Wilson loop operator with no couplings to the scalar. It is basically the same prescription as in [10,11] except that we have Neumann boundary conditions on the $S^{5}$.

1 In other words, despite the great deal of evidence obtained by direct computation of the amplitudes [6,9], we do not know whether this is an exact symmetry of the planar theory. 
In this paper we also consider the problem of computing amplitudes involving the insertion of an operator and an $n$ gluon final state. Such processes arise when we excite the theory and produce final gluon states. We consider the description of these exclusive processes at strong coupling and we will give a prescription for computing them.

This paper is organized as follows. In section two we describe the strong coupling computation of a process involving and operator insertion $\mathcal{O}$ and an $n$ gluon final state. We start with this discussion because the surfaces that appear here will be relevant later, but the casual reader can skip this section. In section three we consider the $n$ gluon scattering amplitude for large $n$ and a very specific kinematic configuration which is chosen so that it approximates a simple spacelike Wilson loop. We use this configuration to test the BDS ansatz [4] at strong coupling and we find a disagreement. In section four we discuss some aspects of the Wilson loop operator with no coupling to the scalars and we perform some weak and strong coupling computations for this Wilson loop. We finally present some conclusions. In appendix A we discuss some aspects of the five gluon solution which we did not find explicitly. In appendix B we give some details for the perturbative computations we did for Wilson loops.

\section{Processes involving a local operator and asymptotic gluons states}

\subsection{Short review on gluon scattering amplitudes at strong coupling}

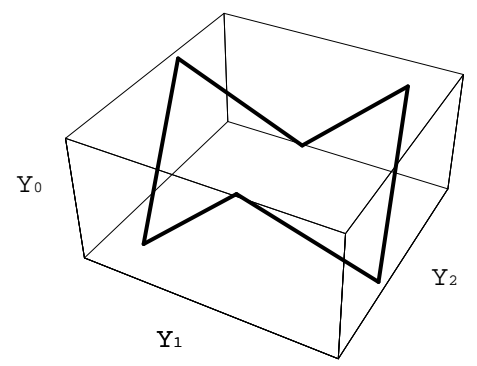

Fig. 1: Sequence of light-like segments on the $A d S_{5}$ boundary given by the set of momenta. The string surface should end on this line at the boundary of $A d S_{5}$. In this case we have six gluons. 
Planar color ordered gluon scattering amplitudes can be computed at strong coupling by considering classical string in $A d S_{5}$ [1]. The amplitude is given in terms of the area of a minimal surface in $A d S_{5}$

$$
\mathcal{A} \sim e^{-\frac{\sqrt{\lambda}}{2 \pi}(\text { Area })}
$$

where we set the radius of curvature of $A d S_{5}$ to one. This is a minimal surface which ends at the boundary of $A d S_{5}$ space on a sequence of lightlike segments given by the fourmomenta of the gluons, see fig. 1. The information about the polarization states of the gluons (or other states of the $\mathcal{N}=4$ super Yang Mills multiplet) comes in at higher orders in the $1 / \sqrt{\lambda}$ expansion. In fact, the next correction to (2.1) is a $\lambda$ independent factor multiplying (2.1). Such a factor could vanish for specific external states. For instance, tree level amplitudes with all helicities identical or all but one identical, vanish due to supersymmetry Ward identities [13].

The $A d S_{5}$ space that appears in the above prescription is parametrized by coordinates $y_{\mu}, \mu=0,1,2,3$ and $r$ with the metric

$$
d s^{2}=\frac{d y^{2}+d r^{2}}{r^{2}}
$$

This space is related to the ordinary $A d S_{5}$ space appearing as the gravity dual of $\mathcal{N}=4$ super Yang Mills by a T-duality along the four worldvolume directions and by $r=1 / z$ where

$$
d s^{2}=\frac{d x^{2}+d z^{2}}{z^{2}}
$$

is the metric of the ordinary $A d S_{5}$ geometry describing the gravity dual of $\mathcal{N}=4$ super Yang Mills and $x^{\mu}$ are the coordinates of the $R^{1,3}$ space where the field theory lives. More details can be found in [1].

\subsection{Processes involving asymptotic gluons and local operators}

In this section we would like to give a prescription for computing amplitudes for processes involving asymptotic gluon states and local operators. For example we can excite the theory by a local operator and look at final states containing a certain number of gluons. We have in mind processes similar to the ones that arise when we consider $e^{+} e^{-} \rightarrow \gamma \rightarrow$ jets. In this case, we can analyze the process to lowest order in $\alpha_{e m}$ but to all orders in $\alpha_{\text {strong }}$ by noticing that the photon couples to the electromagnetic current of QCD and this in turn can produce various final states. Thus the final hadronic state 
is produced by acting with the QCD electromagnetic current on the vacuum. To lowest order in $\alpha_{\text {strong }}$ the state is, of course, a quark anti-quark pair.

We now want to consider analogous processes in $\mathcal{N}=4$ super Yang Mills at strong coupling in the planar approximation. Thus we consider a process where we add a local operator to the theory and we produce gluons. The local operator is a single trace operator with given momentum

$$
\mathcal{O}(q)=\int d^{4} x e^{i q \cdot x} \mathcal{O}(x)
$$

We can consider any operator of the theory. Concrete examples are the stress tensor, the R-symmetry currets, etc.

We are interested in exclusive final states consisting of individual gluons, or other members of the $\mathcal{N}=4$ supermultiplet. From now on the word "gluon" will mean any element of the supermultiplet: a gluon, fermion or scalar, all in the adjoint representation. The asymptotic states for these colored objects are only well defined after we use an IR regulator. The simplest one is dimensional regularization, which consists in going to $4+\epsilon$ dimensions. Then the theory is free in the IR and gluons are good asymptotic states. On the gravity side this can be done by considering the near horizon metrics of D- $p$ branes with $p=3+\epsilon$, as explained in [1].

Once we regularize, we have a worldsheet whose boundary conditions in the far past or future are set at $z \sim \infty$, where the asymptotic gluons live, and the operator conditions are set at the boundary of $A d S_{5}, z \sim 0$, in (2.3). In the T-dual coordinates (2.2) the asymptotic states carry winding number which is proportional to the momentum. The gluon final states are represented as in [1] by considering a sequence of lightlike lines at $r=0$. Each light-like segment joints two points separated by $2 \pi k_{i}^{\mu}$. As opposed to the situation considered in [1], this sequence is not closed. In fact we have $\sum_{i=1}^{n} k_{i}^{\mu}=q^{\mu}$ where $q^{\mu}$ is the momentum of the operator. It is convenient to formally think of the coordinate along $q^{\mu}$ as compact and to consider a closed string as winding that coordinate. This is equivalent to saying that we consider an infinite periodic superposition of the set of momenta $\left\{k_{1}, k_{2}, \cdots, k_{n}\right\}$.

We now should give a prescription for the operator. An operator insertion leads to a string that goes to the $A d S_{5}$ boundary, $z=0$ in the coordinates (2.3). This implies that it should go to $r=\infty$ in the dual coordinates (2.2). Thus we consider a string stretching along the direction $q^{\mu}$ that goes to $r=\infty$.

As a simple example, consider a two gluon state and an operator insertion. The two gluon momenta obey $k_{1}^{\mu}+k_{2}^{\mu}=q^{\mu}$. Let us consider the case where the mometum $q^{\mu}$ is 
spacelike and $k_{1}$ is incoming and $k_{2}$ outgoing. By performing a boost we can choose the momenta as

$$
(2 \pi) k_{1}^{\mu}=\left(\frac{\kappa}{2}, \frac{\kappa}{2}, 0\right), \quad(2 \pi) k_{2}^{\mu}=\left(-\frac{\kappa}{2}, \frac{\kappa}{2}, 0\right), \quad(2 \pi) q^{\mu}=(0, \kappa, 0)
$$

It is convenient to view the direction $y^{1}$ as a compact direction with period $y^{1} \sim y^{1}+\kappa$ so that the total winding number of the string corresponds to an allowed closed string. This closed string has to end on the boundary of the original $A d S_{5}$ space (2.3) at $z=0$. In terms of the dual metric (2.2) it should go to $r=\infty$.
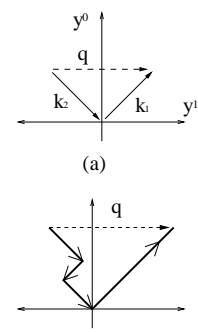

(c)

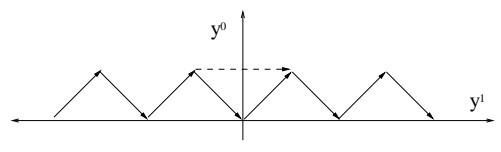

(b)

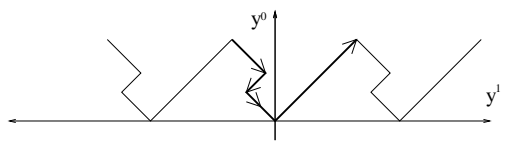

(d)

Fig. 2: (a) We consider the configuration of light-like lines corresponding to the initial and final state gluons under consideration. In (b) we consider an infinite repetition of the configuration. In (c) a more general configuration with four gluons is considered. And in $(\mathrm{d})$ we draw the corresponding periodic version.

In order to find the surface it is convenient to consider an infinite repetition of these momenta, which are following a zig-zag path in the $y^{0}, y^{1}$ plane as shown in figure 2 . We have discussed the solution by thinking of the direction $y_{1}$ as compact. By the time that we undo the T-duality and we go back to the original $A d S_{5}$ coordinates $x, z$ we can forget about the fact that the coordinate is compact. In other words, the final solution, back in $x, z$ coordinates, also describes the case where the $x$ coordinates are non-compact.

We look for a worldsheet which is extended in the radial $A d S_{5}$ direction, from $r=0$, where it ends on the contour displayed in fig. 2(b), and extends all the way to $r \rightarrow \infty$. As we go to large $r$ the surface is extended in the $\hat{1}$ spatial direction but is localized in time. See figure 3 for a picture of the expected surface. 


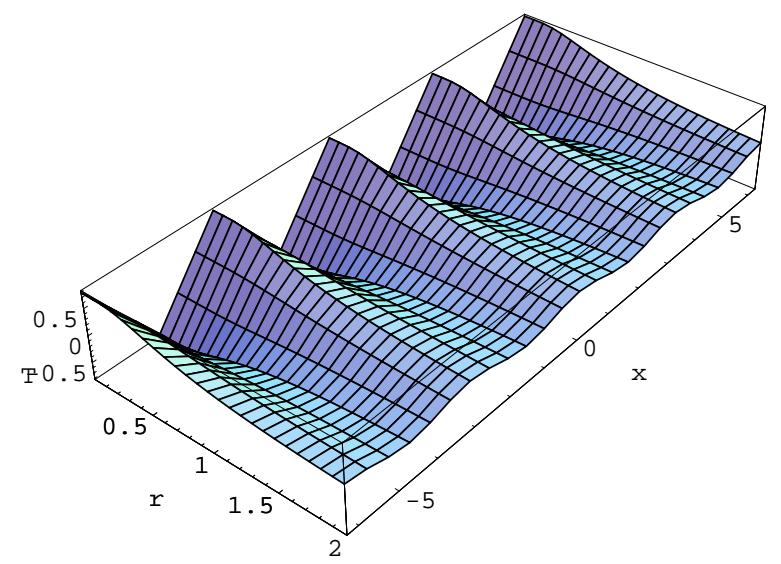

Fig. 3: Approximate form of the solution. At $r=0$ the surface ends on a zig-zag, while for large $r, t$ decays exponentially.

The amplitude is given by computing the area over one period of the resulting surface. Let us point out some features of the solution, which we have not found explicitly. First, one can write the Nambu-Goto action by choosing $r, y$ as worldsheet coordinates so that $t(r, y)$ is the unknown function. The action is

$$
i S=-\frac{R^{2}}{2 \pi \alpha^{\prime}} \int d y d r \frac{\sqrt{1-\left(\partial_{y} t\right)^{2}-\left(\partial_{r} t\right)^{2}}}{r^{2}}
$$

Thus, we should solve the equations of motion that come from this action with the boundary conditions

$$
\begin{aligned}
& \left.t\right|_{r=\infty}=0, \\
& \left.t\right|_{r=0}=y, \quad \text { for } \quad|y| \leq \frac{\kappa}{4}, \quad t_{r=0}=\frac{\kappa}{2}-y, \quad \text { for } \quad \frac{\kappa}{4} \leq y \leq \frac{3 \kappa}{4}
\end{aligned}
$$

and extended in a periodic way outside this range, $t(r, y+\kappa)=t(r, y)$. The second line in (2.7) is simply specifying the path shown in fig. 2(b).

This equation could, in principle, be solved numerically. We expect that for distances much bigger than the size of the zig-zag, i.e. $r \gg \kappa, t(r, y)$ is very small and satisfies a linear equation obtained by expanding (2.6) for small $t$. Expanding $t$ in Fourier modes, $t(r, y)=\sum_{n} t_{n}(r) e^{i k_{n} y}$, with $k_{n}=2 \pi n / \kappa$, we obtain the following equation for $t_{n}(r)$

$$
-k_{n}^{2} t_{n}(r)+r^{2} \partial_{r}\left[\frac{1}{r^{2}} \partial_{r} t_{n}(r)\right]=0 \quad \rightarrow \quad t_{n}(r)=c_{n} e^{-k_{n} r}\left(k_{n} r+1\right)
$$

where we have kept only the decaying solution (for positive $k_{n}$ ). Note that due to the exponential decay, already when $r$ is a few times bigger than $|\kappa|$, the above solution will 
be a good approximation. The coefficients $c_{n}$ are determined by imposing the boundary condition at $r=0$ (2.7), but we should recall that we cannot use the linearized equation in that region. The problem has a scaling symmetry that implies that we can scale out $\kappa$ so that the solution is

$$
t(r, y)=\kappa \hat{t}\left(\frac{r}{\kappa}, \frac{y}{\kappa}\right) \equiv \kappa \hat{t}(\hat{r}, \hat{y})
$$

It is then easy to see, that the value of the classical action (2.6) on this solution is formally independent of the scale $\kappa$, as expected from scaling symmetry. However, since there is a divergence, an explicit $\kappa$ dependence is introduced when we subtract the divergence. Let us understand the divergencies. Let us first consider the large $r$ region. The integral in the region of large $r$ converges since $t \rightarrow 0$ so that we are simply integrating $d r / r^{2}$. This might seem a bit surprising since we expected to obtain terms of the form $\log r$ that are related to the anomalous dimension of the operator. Notice, however, that a logarithmic term in the classical area would have implied an anomalous dimension of order $\sqrt{\lambda}$. Thus, the boundary conditions we considered correspond to operators whose anomalous dimension vanishes at this order. For an operator such as the stress tensor, which has anomalous dimension equal to four, this is indeed the case. We expect to obtain logarithmic terms when we go to higher order in the $1 / \sqrt{\lambda}$ expansion.

We can now consider the small $r$ region. The analysis of this region is the same as the analysis in the small $r$ region for the gluon scattering amplitudes discussed in [1]. One can dimensionally regularize the problem by going to $d=4-2 \epsilon$ dimensions. Then the lagrangian becomes

$$
L=\frac{\sqrt{\lambda}}{2 \pi} c_{\epsilon} \kappa^{-\epsilon} \int d \hat{r} d \hat{y} \hat{r}^{-\epsilon} \mathcal{L}_{0}[\hat{t}(\hat{y}, \hat{r})]
$$

where we have rescaled all variables so that the only dependence on $\kappa$ is in the overall factor. In (2.10) $c_{\epsilon}$ is a function of only $\epsilon$. The divergencies arise from the region near the cusps connecting the momenta of two adjacent gluons (see [四) and they can be computed

2 An example of a configuration where we get an anomalous dimension at leading order is the following. Consider a string winding on the sphere (for example we can replace $S^{5} \rightarrow S^{5} / Z_{k}$ where the $Z_{k}$ acts without fixed points). In that case we will have the action $\frac{\sqrt{\lambda}}{2 \pi} \int \frac{d y d r}{r^{2}} \sqrt{1+r^{2}\left(\partial_{y} \theta\right)^{2}}$. Expanding the square root for $r \rightarrow \infty$ we get $\frac{\sqrt{\lambda}}{2 \pi} \int d y \frac{d r}{r}\left|\partial_{y} \theta\right| \sim \frac{\sqrt{\lambda}}{2 \pi}(\Delta \theta) \log r$. This corresponds to an operator of dimension $\Delta=\frac{\sqrt{\lambda}}{2 \pi} \Delta \theta$ which is indeed what we obtain for a string stretching on the sphere over an angle $\Delta \theta$. 
using the single cusp solution considered in [14,1]. The value of the action is given by integrating only over one period in $y$. It evaluates to a function of the form

$$
i S=-\frac{\sqrt{\lambda}}{2 \pi} \frac{\mu^{\epsilon}}{(2 \pi \kappa)^{\epsilon}}\left[2\left(\frac{1}{\epsilon^{2}}+\frac{1-\log 2}{2 \epsilon}\right)+C\right]
$$

The coefficients of the divergent terms are locally determined and are the same as in [1], so that we would only need the solution to compute the constant $C$. For the simplest case of two gluons, the solution does not depend on any kinematical variable. As we consider configurations with more gluons the solution, and the value of the amplitude, will start depending on the kinematic invariants.

\subsection{Processes involving a mesonic operator and final quark and anti-quarks}

In this subsection we consider a small variant of the configuration considered above. We consider a large $N$ theory with flavors and we insert a mesonic operator, which contains a quark and an antiquark field. Flavors correspond to adding D-branes in the bulk [15, 16]. The mesonic operator corresponds to an open string mode on the D-brane that is extended over $A d S_{5}$. For example, we could consider the insertion of a flavor symmetry current which couples to a $q, \bar{q}$ pair. This is analogous to the electromagnetic current in QCD. Amplitudes involving quarks have been considered at strong coupling in [3]. Once we IR regularize, the quarks correspond to open strings that are attached to the D-brane and sit at $z \sim \infty$ or $r \sim 0$. The discussion is very similar to the one for closed strings. One difference is that now we do not require the configuration to be periodic. However, since we obey Neumann boundary conditions on the boundary of the open string, which translate to Dirichlet boundary conditions in the T-dual variables, we find that the solution can be extended outside the strip into a periodic function with a period which is twice the original width of the strip, see figure 4 .

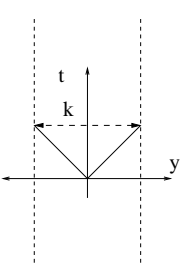

(a)

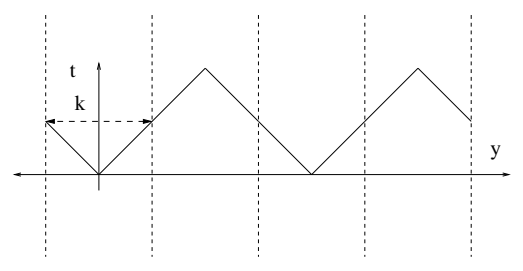

(b)

Fig. 4: Once we extend the solution outside the strip as shown in the figure, it reduces to the zig-zag solution, with twice the period. 
Thus if we consider a configuration with momenta as in (2.5), the solution is simply given by

$$
t=2 \kappa \hat{t}\left(\frac{r}{2 \kappa}, \frac{y}{2 \kappa}\right)
$$

where $\hat{t}$ is the rescaled solution, with period one, $\hat{t}(\hat{r}, \hat{y}+1)=\hat{t}(\hat{r}, \hat{y})$. The action is simply half of the action in (2.11) but with the replacement $\kappa \rightarrow 2 \kappa$. After we reexpress it in terms of $\kappa$ again we get

$$
i S=-\frac{\sqrt{\lambda}}{2 \pi} \frac{\mu^{\epsilon}}{(2 \pi \kappa)^{\epsilon}}\left[\frac{1}{\epsilon^{2}}+\frac{1-3 \log 2}{2 \epsilon}+\left\{\frac{C}{2}-\frac{\log 2}{2}+(\log 2)^{2}\right\}\right]
$$

Thus we see that function $g(\lambda)$ which determines the subleading IR divergencies is different for a gluon than a quark. Namely, we have

$$
g_{\text {gluon }}(\lambda)=\frac{\sqrt{\lambda}}{2 \pi}(1-\log 2), \quad g_{\text {quark }}(\lambda)=\frac{\sqrt{\lambda}}{2 \pi}(1-3 \log 2)
$$

where $g_{\text {gluon }}$ as computed in [1]. In the case that we have a cusp that joins a quark and a gluon we expect to have the average of the above two formulas.

We can similarly consider asymptotic states corresponding to a quark and an antiquark plus extra gluons, $q \bar{q}+n g$. In this case we simply take the configuration of momenta for all these particles, we flip it, and then take a periodic superposition as explained above. See figure 5. The solution will be then given by half of the periodic solution as we had above.
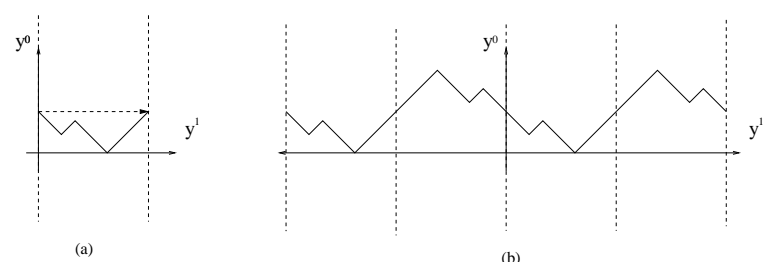

Fig. 5: (a) Configuration of momenta for the process $\gamma \rightarrow q \bar{q}+g+g$. The dashed line with an arrow indicates the momentum of the operator. The first and last segments are the quark and anti-quark, the middle two segments are the two gluons. (b) Extension of the momenta into a periodic configuration.

\section{Scattering amplitudes involving a large number of gluons}

\subsection{The Bern Dixon Smirnov ansatz}

In an interesting paper, Bern, Dixon and Smirnov [4], proposed the following form for the $n$ gluon planar, color ordered, MHV scattering amplitude

$$
\log \left[\frac{\mathcal{A}_{n}}{\mathcal{A}_{n, \text { Tree }}}\right]=\operatorname{Div}_{n}+\frac{f(\lambda)}{4} a_{1}\left(k_{1}, \cdots, k_{n}\right)+h(\lambda)+n k(\lambda)
$$


where $a_{1}$ is the finite part of the one loop scattering amplitude, up to a factor of $\frac{\lambda}{8 \pi^{2}}$. The divergent terms have a simple structure controlled by the cusp anomalous dimension $f(\lambda)$ and a second function of the coupling $g(\lambda)$. In dimensional regularization, $d=4-2 \epsilon$, we have 4

$$
\begin{gathered}
\operatorname{Div}_{n}=-\sum_{i=1}^{n}\left[\frac{1}{8 \epsilon^{2}} f^{(-2)}\left(\frac{\lambda \mu^{2 \epsilon}}{s_{i, i+1}^{\epsilon}}\right)+\frac{1}{4 \epsilon} g^{(-1)}\left(\frac{\lambda \mu^{2 \epsilon}}{s_{i, i+1}^{\epsilon}}\right)\right] \\
\left(\lambda \frac{d}{d \lambda}\right)^{2} f^{(-2)}(\lambda)=f(\lambda), \quad \lambda \frac{d}{d \lambda} g^{(-1)}(\lambda)=g(\lambda)
\end{gathered}
$$

where $\mu=\mu_{I R}$ is the IR regularization scale. $h(\lambda)$ and $k(\lambda)$ are functions of only $\lambda$ and will not be interesting for us. We will focus on $a_{1}$ which is the part of the amplitude that depends in a non-trivial way on the kinematic invariants of the process. Note that $f(\lambda)$ is a known function [17] which behaves as [18,19], see also [20,

$$
f(\lambda)=\left\{\begin{array}{l}
\frac{\lambda}{2 \pi^{2}}\left(1-\frac{\lambda}{48}+\cdots\right), \quad \text { for } \quad \lambda \ll 1 \\
\frac{\sqrt{\lambda}}{\pi}+\cdots, \quad \text { for } \quad \lambda \gg 1
\end{array}\right.
$$

The results for four and five gluons $(n=4,5)$ are determined by the momentum space (or T-dual) conformal symmetry [7,5]. Thus, their form follows from the form of the leading IR divergence which is controlled by the function $f(\lambda)$. Thus, in order to do a non-trivial test of the BDS guess we need to perform a higher loop computation for $n \geq 6$, where the results are not fixed by the dual conformal symmetry. In what follows below, we will do a computation at large $\lambda$ for large $n$ and we will find a disagreement with (3.1).

In a recent paper Brandhuber et al [12], have shown that the finite part of the one loop scattering amplitude $a_{1}\left(k_{1}, \cdots, k_{n}\right)$ is equal to the finite part of the one loop expectation value of a Wilson loop consisting of $n$ lightlike segments specified by the momenta (for $n=4$ this was shown in [7]). This relation has been verified explicitly by computing both sides and checking that they are the same. In other words, we consider a Wilson loop specified by light-like segments proportional to the momenta $k_{i}^{\mu}$. We consider its expectation value to obtain

$$
\left\langle W_{\left\{k_{i}\right\}}\right\rangle=1+\frac{\lambda}{8 \pi^{2}}\left[\operatorname{Div}^{\prime}+w_{1}\left(k_{1}, \cdots, k_{n}\right)+c+n c^{\prime}\right]
$$

Then the result of [12] is that $w_{1}\left(k_{1}, \cdots, k_{n}\right)=a_{1}\left(k_{1}, \cdots, k_{n}\right)$. The divergent terms arise from UV divergencies at the cusps of the Wilson lines. They have a form similar to (3.2) 
but with $\mu_{I R} \rightarrow \mu_{U V}$ and with a different function $g(\lambda)$ [7] [12]. Thus we can say that the BDS ansatz is simply saying that the $n$ gluon scattering amplitude is the same as the exponentiation, up to the function $f(\lambda)$, of the one loop expectation value of a Wilson loop. In other words, we can write

$$
\left.\frac{\mathcal{A}}{\mathcal{A}_{\text {Tree }}}\right|_{B D S}=e^{\operatorname{Div}} e^{\frac{f(\lambda)}{4} w_{1}\left(k_{1}, \cdots, k_{n}\right)} e^{\tilde{h}(\lambda)+n \tilde{k}(\lambda)}
$$

where $\tilde{h}(\lambda)$ and $\tilde{k}(\lambda)$ are functions which depend only on $\lambda$ but are independent of the shape of the Wilson loop and will not be interesting for us.

We now consider the strong coupling form of the amplitude. After we go to the Tdual AdS space we find that the computation is formally equivalent to computing the expectation value of a Wilson loop, at least to leading order in the $1 / \sqrt{\lambda}$ expansion. On the other hand we know that the strong coupling result for a general Wilson loop is not simply given by exponentiating the one loop value and multiplying by the function $f(\lambda)$. In particular, let us recall the expectation value for a spacelike rectangular Wilson loop with long length $T$ and short length $L$, with $T / L \gg 1$. This is the Wilson loop that is useful for computing the quark anti-quark potential. Sometimes one considers the long side along a timelike direction. Here we consider both sides of the rectangle along spacelike directions, so that time is orthogonal to the loop.

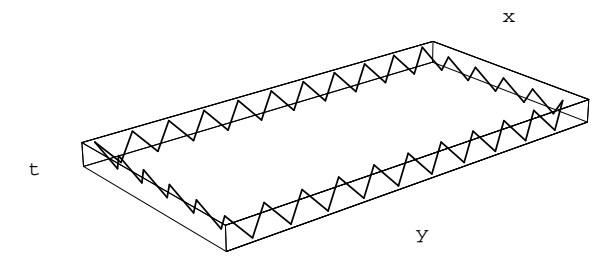

Fig. 6: Configuration of gluons approximating a rectangular Wilson loop. Here $n=60$ and $T=2 L$. The rectangle lies along two spatial dimensions and the zig-zag motion is into the time direction.

On the other hand, the Wilson loops that appear in amplitude computations consist of light-like segments that are are not obviously related to the rectangular spacelike Wilson loop. However, one can approximate a spacelike Wilson loop via a Wilson loop with lightlike segments. For instance, one can approximate a straight spacelike line with a zigzag path of lightlike segments, such as the one shown in fig. 2(b). Thus, we can approximate the rectangular Wilson loop via the configuration of light-like lines shown in figure fig. 6 . 
If the zig-zag segments have spatial length $\kappa$, then we expect that at distances longer than $\kappa$ the loop will look like a spacelike one. In fact, in the leading weak coupling approximation, $w_{1}$, this is very simple to see, since the one loop approximation to the Wilson loop is given by integrating the propagator between two points along the loop. As long as the two points are far away the zig-zag motion averages out and we recover the same result as for a spacelike loop, up to terms that can be viewed as the local divergencies that appear when we consider a spacelike loop. Note that any two points on this zigzag loop that are not on the same segment are spacelike separated. In addition, note that the spacelike Wilson loop that we get in the limit is the one with no scalar couplings, namely the ordinary Wilson loop operator $W=\operatorname{Tr}\left[P e^{i \oint A}\right]$, we will return to this point later. The weak coupling result for the rectangular loop, for $T / L \gg 1$, is

$$
\log \langle W\rangle=\frac{\lambda}{8 \pi} \frac{T}{L}\left(1-\frac{\lambda}{4 \pi^{2}}+\cdots\right), \quad \lambda \ll 1
$$

where the two loops term is computed in appendix B. Note that the coefficient of the one loop term is half of the value for the locally BPS Wilson loop that contains a coupling to a scalar3.

We now consider the configuration at strong coupling. Near the zig-zag path the solution will look like the solution discussed in section 2, see fig. 3. As we move along the radial $A d S_{5}$ direction into the bulk the surface approaches very quickly the solution we would have for a straight spacelike Wilson loop, see the discussion around (2.8). More quantitatively, if we introduce a zig-zag path with segments of spatial length $\kappa / 2$ the classical area behaves as

$$
\text { Area }=\frac{\ell}{\kappa} \frac{\mu^{\epsilon}}{(2 \pi \kappa)^{\epsilon}}\left(\frac{2}{\epsilon^{2}}+\frac{(1-\log 2)}{\epsilon}+C\right)+A_{\text {ren }}+\cdots
$$

where $\ell$ is the length of the spacelike loop that we are approximating. The constant $C$ is the same as in (2.11), but its value is not important for us. Finally, $A_{\text {ren }}$ is the value we would have for a spacelike Wilson loop. Namely, for a spacelike Wilson loop we have

$$
\text { Area }=\frac{\ell}{a}+A_{\text {ren }}
$$

where we have set a cutoff at $z=a$ and taken the limit $a \rightarrow 0$ to define $A_{\text {ren }}$. We see that after we subtract the terms going like $1 / \epsilon^{2}$ and $1 / \epsilon$ which are the IR divergencies in the

3 The two loop term was computed for the locally BPS Wilson loop in [21], see also 22]. 
amplitude in (3.7) we are left with a term that is finite in the $\kappa \rightarrow 0$ limit, $A_{\text {ren }}$, and a term that diverges, proportional to $C$. This term, which goes like $\frac{\ell}{\kappa} C$, can be interpreted as the local divergence that appears when we define a spacelike Wilson loop, see (3.8). This divergence is non-universal and could depend on how we define the spacelike Wilson loop. This divergent term is proportional to the perimeter length of the loop and does not depend on the macroscopic shape of the loop. In the particular case of the rectangular loop, the divergent term does not depend on $T / L$. Therefore we can subtract it to obtain a finite value, $A_{\text {ren }}$, which should coincide with the finite value of the spatial Wilson loop expectation value. More precisely, we get $\langle W\rangle \sim e^{-\frac{\sqrt{\lambda}}{2 \pi} A_{\text {ren }}}$ to leading order in $1 / \sqrt{\lambda}$.

Since the light like lines had no coupling to the scalars, the Wilson loop that we approximate in this way is the Wilson loop with no scalar coupling. However, as we show in section 4 , to leading order in the $1 / \sqrt{\lambda}$ expansion, the two results are the same. Both are given in terms of the same minimal surface in $A d S_{5}$. Then we find that the result for the rectangular loop in the regime $T / L \gg 1$ is given by [10,11],

$$
\log \langle W\rangle=\sqrt{\lambda} \frac{4 \pi^{2}}{\Gamma\left(\frac{1}{4}\right)^{4}} \frac{T}{L}, \quad \lambda \gg 1
$$

The final conclusion is that for a particular configuration of gluons, where $n \rightarrow \infty$ we can approximate both the BDS guess and the large $\lambda$ results by considering a spacelike loop. In this limit, if the BDS result were true, we would obtain a different numerical coefficient for the result of a Wilson loop expectation value. Instead of the second line in (3.9) the BDS guess would produce $\log \langle W\rangle=\frac{\sqrt{\lambda}}{4} \frac{T}{L}$, were we used the known strong coupling form for the function $f(\lambda)$ (3.3). We should also mention that the functions $h(\lambda)$ and $k(\lambda)$ that appear in (3.1) cannot fix the disagreement since they are independent of the shape of the Wilson loop.

The reader might worry about the following. The strong coupling prescription for the Wilson loops that we are using is simply the leading order term in the $\sqrt{\lambda}$ expansion. At the next order we can have further fields that propagate on the worldsheet that encode the polarization states of the asymptotic states. Since we are taking the $n \rightarrow \infty$ limit, one might worry that such terms might become important. In particular, we have not used the fact that we are considering an MHV amplitude. In order to avoid this problem we take the large $\lambda$ limit and then the large $n$ limit, so that $\sqrt{\lambda} \gg n \rightarrow \infty$. With this particular order of limits we do not have to worry about such subleading corrections. We can take the same limit of the BDS guess [4], which was supposed to be valid for all values of $\lambda$ and $n$.

Of course, it would be nice to find out exactly at what order in $\lambda$ the first deviation appears. For this purpose we should consider an amplitude for $n \geq 6$ external particles. 


\subsection{Scattering amplitudes and Wilson loops}

Recently, another conjecture has been entertained [7, 12,5], which agrees with what we know presently. The conjecture is that the finite part of the planar color ordered MHV amplitude is given by the finite part of the Wilson loop expectation value in the full theory

$$
\frac{\mathcal{A}_{n}}{\mathcal{A}_{n, \text { Tree }}}=e^{\text {Div }}\left\langle W_{k_{i}}\right\rangle e^{-D i v^{\prime}} e^{\hat{h}(\lambda)+n \hat{k}(\lambda)}
$$

Where Div corresponds to the IR divergencies of the amplitude, while Div' is subtracting the UV divergencies of the Wilson loop. The functions $\hat{h}(\lambda)$ and $\hat{k}(\lambda)$ are independent of the shape of the Wilson loop. The statement is only about the momentum dependent finite remainder of the amplitude. Of course, this does not give us an explicit function since we do not know how to independently compute the expectation value of the Wilson loop.

From the string theory point of view this conjecture implies that computing the Wilson loop expectation value in the T-dual $A d S_{5}$ space gives the same answer as computing it in the original $A d S_{5}$ space. At leading order in the $1 / \sqrt{\lambda}$ expansion, this is obvious since all we do in both cases is to compute the area of the minimal surface in $A d S_{5}$. On the other hand, to higher orders in the $1 / \sqrt{\lambda}$ expansion we would start noticing that the RR fields and the dilaton are different in both cases. Thus, the conjecture amounts to the statement that we can perform a redefinition of the worldsheet fermions in such a way that the two backgrounds lead to the same result. It would be interesting to check explicitly whether this is true or not. Note that if the "dual" momentum space conformal symmetry [6] is an exact symmetry of the theory, then the agreement between Wilson loops and amplitudes for $n=4,5$ would be obvious since in both cases the answer is determined by the symmetries [7, 5].

As another remark in the same direction, we note that the two loop result for the rectangular Wilson loop at weak coupling, (3.6), is not given by the two loop correction to the function $f(\lambda)(3.3)$. This implies that the two loop expression for the Wilson loop is not given by the exponential of the one loop result (up to the function $f(\lambda)$ ) for $n$ large. In turn, this implies that either the relation between amplitudes and Wilson loops is not correct at two loops or that the BDS guess for the amplitude is not correct at two loops 4 . In any case, we see that we would learn something from an explicit two loop computation of the amplitude for $n \geq 6$.

4 It could well be that the BDS guess for the amplitude is correct at two loops for all $n$, but that the relation between Wilson loops and the amplitudes is incorrect at two loops. 


\section{String dual of the ordinary Wilson loop}

In this section we discuss our proposal for the string dual of the ordinary Wilson loop operator

$$
W(\mathcal{C})=\frac{1}{N} \operatorname{Tr}\left[P e^{i \oint_{\mathcal{C}} \dot{x}^{\mu} A_{\mu}}\right]
$$

Note that in $\mathcal{N}=4$ super Yang Mills one often considers the locally 1/2 BPS Wilson loop which contains a coupling to scalar fields

$$
W_{B P S}(\mathcal{C})=\frac{1}{N} \operatorname{Tr}\left[P e^{\oint_{\mathcal{C}} i \dot{x}^{\mu} A_{\mu}+|\dot{x}| \theta^{i} \Phi^{i}}\right]
$$

where $\phi^{i}$ are the six scalar fields of $\mathcal{N}=4 \mathrm{SYM}$ and $\theta^{i}$ a unit vector in $R^{6}$. At strong coupling we know that the description of the Wilson loop (4.2) is given by a string worldsheet that ends on the boundary of $A d S_{5}$ on the corresponding contour with Dirichlet boundary conditions on the sphere which force the loop to sit at the point $\theta^{i}$ [10,11, 23].

The above discussion suggests that we can approximate a Wilson loop with no coupling to the scalars, such as (4.1) by a ziz-zag Wilson loop. Note that the light-like Wilson loops do not have couplings to the scalars.

This leads to a natural conjecture for the string dual of the ordinary Wilson loop. Namely, the ordinary Wilson loop operator, (4.1), is described by a string worldsheet that ends on the boundary of $A d S$ on the loop $\mathcal{C}$ and has Neumann boundary conditions on the five-sphere. The idea that this loop is somehow related to Neumann conditions also appeared in [23], but an explicit prescription was not stated.

We make a simple consistency check of this relation. Let us consider the gauge theory on $S^{3} \times R$ and consider a Wilson line for a quark sitting at a point on the $S^{3}$ and an antiquark at the opposite point. At strong coupling such loop corresponds to a worldsheet which is $A d S_{2}$ and is embedded in $A d S_{5}$. One can check that it costs finite energy to move the position of the string on the $S^{5}$. It is consistent with the equations of motion of the string to set Neumann boundary conditions. In fact, if we consider small fluctuations on the $S^{5}$ we see that these are described, to leading order, by massless fields that live on $A d S_{2}$. In other words, we have massless fields living on a strip of length $\pi$. Thus, the dimension of operators with spin $J$ in $S O(6)$ is given by

$$
\Delta=\frac{J(J+4)}{\sqrt{\lambda}}+o\left(\frac{1}{\lambda}\right)
$$

which arises simply by considering the quantum mechanics of the center of mass motion, which is the lowest mode on the strip, when we have Neumann boundary conditions. 
We see that we get finite energies for states whose endpoints are moving on the $S^{5}$. In fact, if we consider the simplest case, with $J=1$, we see that the energy (4.3) can be interpreted as the anomalous dimension, at strong coupling, for the insertion of an operator $\phi^{i}$ along the loop. Namely, we consider an operator of the form

$$
P e^{i \int_{-\infty}^{0} A} \phi^{i}(0) P e^{i \int_{0}^{\infty} A}
$$

If we consider a straight contour which preserves an $S L(2)$ subgroup of the conformal group which includes the dilatation operator, then we see that we can assign well defined scaling dimensions to such operators along a Wilson loop. These scaling dimensions then determine the correlation functions for two insertions. In general, when we have two insertions of such operators we have

$$
\left\langle P\left[\mathcal{O}(0) \mathcal{O}(a) e^{i \int A}\right]\right\rangle=\frac{C}{a^{2 \Delta}}\left\langle P e^{i \int A}\right\rangle
$$

Using standard perturbation theory we can compute the leading correction to the anomalous dimension for the $\phi^{i}$ insertion and we obtain (see appendix B for more details)

$$
\Delta=1-\frac{\lambda}{8 \pi^{2}}+o\left(\lambda^{2}\right)
$$

Thus we see that already to leading order in the weak coupling expansion the anomalous dimension tends to go down, and we observe that at strong coupling this anomalous dimension is close to zero since it goes like $1 / \sqrt{\lambda}$.

This should be contrasted with the results that are obtained for the $1 / 2$ BPS Wilson loop. Let us say that we consider a loop which couples to the operator $\phi^{6}$ (the operator (4.2) with $\theta^{6}=1$ ). Then the insertion of the operators $\phi^{1}, \cdots, \phi^{5}$ corresponds to BPS operators of dimension $\Delta=1$, see the discussion in [24]. On the other hand the dimension of insertions of $\phi^{6}$ do not correspond to any obvious light field on the string worldsheet 5 . Thus it is natural to think that at strong coupling its dimension will be of the order of the mass of the typical string state which goes like $\lambda^{1 / 4}$ as in 25]. Indeed, when we compute the weak coupling anomalous dimension of this operator we find that it is not protected with the following anomalous dimension

$$
\Delta=1+\frac{\lambda}{4 \pi^{2}}+o\left(\lambda^{2}\right)
$$

5 We thank L. Yaffe for asking us this question. 
So we see that quantum corrections increase the anomalous dimension. Presumably this continues to increase so that at strong coupling it has a value larger than $\lambda^{1 / 4}$.

Notice that, to leading order, the strong coupling result for the Wilson loop is the same as for the locally supersymmetric Wilson loop with a constant $\theta^{i}$. However, we will

find differences at the next order in the $1 / \sqrt{\lambda}$ expansion since in the case of the ordinary loop we would have to integrate over the point on the sphere where the string is sitting. More precisely, at the one loop level in the $\alpha^{\prime}$ expansion we find that the determinants for quadratic fluctuations are different in the two cases [26].

On the other hand, the weak coupling result is rather different for the two loops. For example the leading term in the quark anti-quark potential for the ordinary Wilson loop is half the value we have for the locally half BPS loop since we only have the gauge boson exchange, rather than the boson exchange plus the scalar exchange. In fact, we also computed the two loop correction for the quark anti-quark potential and we found the result shown in (3.6). The two loop correction for the quark anti-quark potential coming from the half BPS Wilson loop was computed in [21,22], where a logarithmic term of the form $\log \lambda$ was found at this order.

\section{Conclusions}

In this article we have given a prescription for computing processes involving local operators and single gluon asymptotic states at strong coupling in $\mathcal{N}=4$ super Yang Mills. As in the case where we only have gluons, the problem reduces to finding a classical surface in a T-dual $A d S_{5}$ space. The surface ends on a contour on the boundary of this T-dual AdS space which is specified by the momenta of the gluons. In addition the surface also goes to $r \rightarrow \infty$ which corresponds to the boundary of the original $A d S_{5}$ space. This boundary condition characterizes the operator in question.

We have then proceeded to test the BDS [1] ansatz for a particular configuration involving a large number of gluons $n \rightarrow \infty$. We found that it did not survive the test. We took the $n \rightarrow \infty$ limit to simplify the computations. However, we think that it is likely that already for $n=6$ the strong coupling answer will not have the BDS form [4].

On the other hand, recently [7, 12,5] an interesting equivalence between two seemingly different observables was considered: scattering amplitudes and Wilson loops. Our results are consistent with such relation. However, our weak coupling computation for the rectangular Wilson loop suggests that something new is happening at two loops in the 
weak coupling expansion. We found that the Wilson loop vacuum expectation value is not given by the BDS ansatz. The reason could be that the Wilson loop is not equal to the amplitude or that the BDS ansatz is not the correct value of the amplitude at two loops. So, we would learn something new by computing the amplitude at two loops for $n \geq 6$.

We have also considered some features of the ordinary Wilson loop in $\mathcal{N}=4$ super Yang Mills. This is the Wilson loop with no scalar couplings. We described the string prescription that corresponds to this Wilson loop. We have also computed the anomalous dimension for the insertion of a scalar field at a point on this Wilson loop, both at strong and weak coupling. We have also computed the static potential for the ordinary Wilson loop to two loops. This result does not satisfy the exponentiation properties suggested by the BDS conjecture. As mentioned above, this suggest that either the BDS conjecture or the relation between Wilson loops and scattering amplitudes fails at two loops for a large enough number of gluons.

\section{Acknowledgments}

We would like to thank Z. Bern, L. Dixon, G. Korchemsky, H. Liu, J. McGreevy, G. Sterman, E. Sokatchev, M. Spradlin, A. Volovich and L. Yaffe for discussions.

This work was supported in part by U.S. Department of Energy grant \#DE-FG0290ER40542. The work of L.F.A was supported by VENI grant 680-47-113.

\section{Appendix A. Some remarks on the worldsheet solution for five gluons.}

At strong coupling, the amplitude is computed by consider a Wilson loop with five light-like segments that end on five points. The data specifying the configuration corresponds to the five points $x_{1}, \cdots, x_{5}$ which are such that $\left(x_{i}-x_{i+1}\right)^{2}=0$. One can easily check that there are no invariants that can be made out of these five points. In particular, by a conformal transformation we can always map points $x_{1} \rightarrow 0$ and $x_{2} \rightarrow(1,1, \overrightarrow{0})$. Then the other three points are on the light-like boundary of Minkowski space. Another way to see this is that we have $4 \times 5-5=15$ parameters and this is the same as the dimension of the conformal group. This argument also shows that when we go to $n=6$ we expect that conformal symmetry will not determine the answer.

As in the case of $n=4$ [7] one can show that the $n=5$ Wilson loop is completely

determined by conformal symmetry6 [5]. Of course, the fact that we get a non-trivial

6 We thank M. Spradlin and A. Volovich for a conversation on these issues. 
function comes from the fact that we have divergencies at the cusps, otherwise we would get a result that is independent of the momenta. Thus we do not need to find the explicit surface to know that the strong coupling answer will agree with the BDS conjecture [4], for $n=5$. Nevertheless let us make a few remarks on finding the surface explicitly. The surface is simplest to understand if we have a spacelike momentum transfer at all the cusps. Other cases could be found by an appropriate analytic continuation.

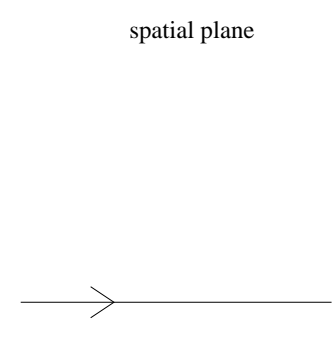

(a) timelike plane $\quad \mathrm{X}_{1}^{ \pm}$plane

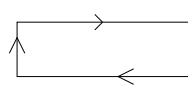

(b)

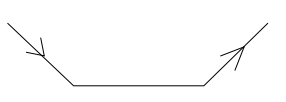

(c)

Fig. 7: Wilson loop in $R^{2,2}$. We display the contours in the $\left(x_{1}, x_{2}\right),\left(t_{1}, t_{2}\right)$ and $\left(x_{1}^{+}, x_{1}^{-}\right)$plane respectively of a simple configuration corresponding to the scattering of 5 gluons. The remaining three points are located on the light-like boundary of $R^{2,2}$.

In $1+3$ signature it is not possible to have a real configuration of five light-like momenta so that the sum of two consecutive ones is always spacelike. On the other hand this is possible for real momenta in $2+2$ signature. This corresponds to complex momenta in $1+2$ signature. Thus it is convenient to look for the surface using $2+2$ signature, where there is a completely real solution. Since all configurations are related by conformal symmetry, we can attempt to find the simplest one. A simple configuration is the one shown in fig. 7. It consists of a set of three lines in $R^{2,2}$, the other two lines live on the light-like boundary of $R^{2,2}$.7 The three lines are parametrized by $\left(t_{1}, t_{2}, x_{1}, x_{2}\right)$ given by

$$
\begin{aligned}
& (\lambda,-1,-\lambda, 0), \quad \lambda \geq 1 \\
& (1, \lambda, \lambda, 0), \quad-1 \leq \lambda \leq 1 \\
& (\lambda, 1, \lambda, 0), \quad \lambda \geq 1
\end{aligned}
$$

7 One can relate $R^{2,2}$ to a patch of $S^{2} \times S^{2}$ in the same way that $R^{1,3}$ is embedded into a patch of $R \times S^{3}$. This is done as follows. We write $d s^{2}=-d r_{1}^{2}-r_{1}^{2} d \varphi_{1}^{2}+d r_{2}^{2}+r_{2}^{2} d \varphi_{2}^{2}=$ $\frac{1}{4 \cos ^{2} v^{+} \cos ^{2} v^{-}}\left(-d \theta_{1}^{2}-\sin ^{2} \theta_{1} d \varphi_{1}^{2}+d \theta_{2}^{2}+\sin ^{2} \theta_{2} d \varphi_{2}^{2}\right)$, where $r_{1} \pm r_{2}=\tan v^{ \pm}$and $v^{ \pm}=\left(\theta_{1} \pm \theta_{2}\right) / 2$. 
We can see that $x_{2}=0$ everywhere and we can view the solution as $t_{2}\left(x_{1}^{+}, x_{1}^{-}\right)$where

$x_{1}^{ \pm}=t_{1} \pm x_{1}$. The behavior near each of the cusps is given by the single cusp solution in [14]. In order to gain some intuition of this solution, we can study the equations of motion in the regime of large $x_{1}^{ \pm}$. In this regime $t_{2}$ is small and we can linearize the equation

$$
\left(\partial_{\tau}^{2}+\partial_{\chi}^{2}-2 \partial_{\tau}\right) t_{2}(\chi, \tau)=0
$$

where $x^{ \pm}=e^{v^{ \pm}}$and $v^{ \pm}=\tau \pm \chi$. It seems natural to assume that for large $\tau$ the function behaves as $t_{2}=t_{2}(\chi / \sqrt{\tau})$ and assume that $u=\chi / \sqrt{\tau}$ is kept fixed as $\tau$ becomes large. Then we find the following form for the solution

$$
u \partial_{u} t_{2}(u)+\partial_{u}^{2} t_{2}(u)=0, \quad \rightarrow \quad t_{2}(u) \approx \operatorname{Erf}(u / \sqrt{2})
$$

The presence of the error function suggest that the full solution may not have a simple algebraic expression.

\section{Appendix B. Some perturbative computations involving Wilson loops}

\section{B.1. One loop anomalous dimensions of scalar insertions}

We normalize the action so that there is an overall $1 / g^{2}$ term. Then the propagator of the scalar fields has the form

$$
\left\langle\phi_{i}^{j}(x) \phi_{k}^{l}(0)\right\rangle=\frac{g^{2}}{8 \pi^{2}} \frac{\delta_{i}^{l} \delta_{k}^{j}}{x^{2}}
$$

and $\lambda=g^{2} N$.

Let us first consider the one loop computation of anomalous dimensions for local operators inserted on the loop. We compute them by considering the correlation function

$$
\left\langle P\left[\mathcal{O}(0) \mathcal{O}(a) e^{i \int A}\right]\right\rangle=\frac{C}{a^{2 \Delta}}\left\langle P e^{i \int A}\right\rangle
$$

where $C$ is a constant and $P$ denotes ordering along the path. Let us now compute the anomalous dimension for an insertion of an $S O(6)$ scalar $\phi^{i}$ along the ordinary Wilson loop (4.1). At lowest order we simply have the contribution in fig. 8(c). At the next order in $\lambda$ we get several diagrams. In order to simplify the computation we note that very similar diagrams appear when one considers the correction to the anomalous dimension for an insertion of $\phi^{1}$ into a BPS Wilson loop which involves $\phi^{6}$, such at (4.2) with $\theta^{6}=1$. 


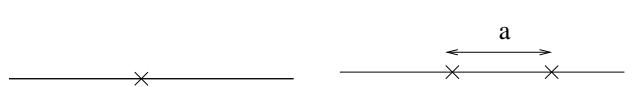

(a)

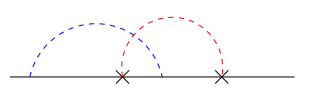

(d)

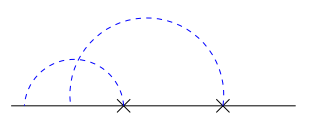

(f)

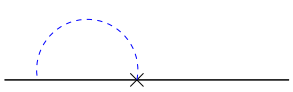

(i) (b)

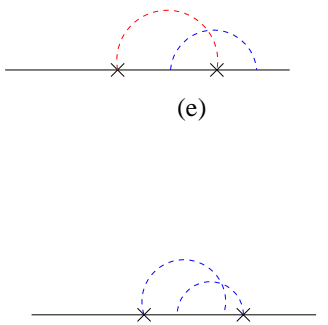

(g)

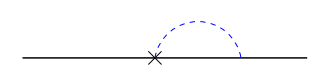

(j)

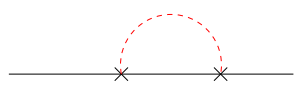

(c)

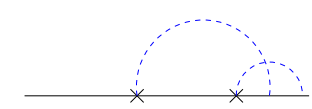

(h)

Fig. 8: In (a) we see a local operator insertion along a Wilson loop. In (b) we see two operators insertions leading to a two point function. In (c) we see the tree level contribution to the two point function. In (d),(e) we see diagrams that determine the contribution of the anomalous dimension of a $\phi^{1}$ insertion along an ordinary Wilson loop. These are diagrams that end up contributing (with a minus sign) to the BPS wilson loop. Thus, they contribute with a plus sign to the ordinary loop, as explained above. In (f),(g),(h) we see various diagrams that contribute to the two point function of the operator $\phi^{6}$ which also appears in the expression of the BPS Wilson loop. These diagrams contribute with minus signs because they represent the contributions that we oversubtract when we subtract the exponentiation of the diagrams in (i),(j) which would lead to a one point function.

For the BPS loop the $\phi^{1}$ insertion is BPS and its anomalous dimension is protected. Thus, the only diagrams that can contribute to the anomalous dimension in our case come from diagrams which appear for the BPS loop but do not appear for the ordinary loop or viceversa. The only such diagrams are diagrams involving contractions of the scalar field $\phi^{6}$ which appears in the exponent of the BPS Wilson loop. At this order we do not have contractions between the inserted field, $\phi^{1}$, and the field appearing in the exponent, $\phi^{6}$. However, the inserted field affects the contractions of the scalar that appears in the loop because of the planarity restriction. Note that those contractions also appear in the right hand side of $(\overline{B .2})$ when we compute the expectation value of the loop without an insertion. Thus we need to consider diagrams which represent contributions that are in 
the right hand of the BPS loop side but are not in the left hand side of the BPS loop, due to planarity. Such diagrams are shown in figure fig. 8(d),(e). These diagrams contribute to a term of the form

$$
\left\langle P \phi^{i}(0) \phi^{i}(a) e^{i \int A}\right\rangle=\frac{C}{a^{2}}\left(1+\frac{\lambda^{2}}{4 \pi^{2}} \log (a / \epsilon)\right)\left\langle e^{i \int A}\right\rangle
$$

This implies that the anomalous dimension is (4.6).

In a similar way we can compute the anomalous dimension for the insertion of a $\phi^{6}$ field on a half BPS Wilson loop which couples to $\phi^{6}$ (i.e. (4.2) with $\theta^{6}=1$ ). This operator will have a non-zero expectation value $\langle\phi\rangle$ which will come from contractions between the insertion and the fields along the loop, see fig. 8(i),(j). These should be subtracted to define a good conformal operator, whose expectation value is zero. So from now on we consider the operator defined with these subtractions. As before, we only have to consider the diagrams which appear when we insert a $\phi^{6}$ but do not appear when we insert a $\phi^{1}$ or viceversa. Such diagrams are the ones where the insertion is contracted with the fields appearing in the loop. There are many diagrams of this kind. However, all the planar diagrams are subtracted when we subtract the vacuum expectation value of $\phi^{1}$ to define the operator. In fact, this subtraction is subtracting diagrams which do not appear when we consider the two point function without any subtraction. These extra subtractions are the only contribution and are displayed in fig. 8(f),(g),(h). They thus contribute with a minus sign. Computing explicitly such diagrams one focuses on the term going like $\log a$ from which we can extract the correction to the anomalous dimension to obtain (4.7).

\section{B.2. Two loop computation of the quark antiquark potential}

In this appendix we give some details on the computation of the quark anti-quark potential for the case that we have a quark that couples only to the gauge field, as in (4.1). A similar computation for the case that we also have a scalar coupling was done in [21,22]. We perform the computation in Euclidean space. 


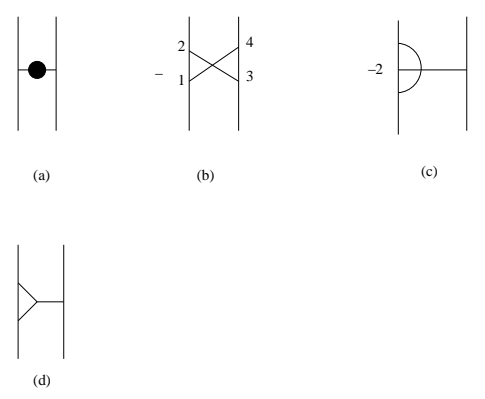

Fig. 9: All lines, except the vertical ones, are gluon propagators. (a) One loop vacuum polarization correction to the gluon propagator. (b) Non iterative ladder. This diagram represents the failure the exponentiation of the one loop result, so it should be subtracted from the log of the Wilson loop expectation value. (c) Similar non-iterative subtraction. The factor of two because it appears on both lines. Note that the lines that cross in (b) and (c) do not denote interaction vertices. (d) Diagrams with an interaction vertex that does not contribute.

The diagrams to be computed as are in figure fig. 9. The diagram in fig. 9(d) does not contribute in Feynman gauge because all the indices of the gluons along the loop are all forced to zero 0, and there is no vertex in the Yang Mills lagrangian involving three gauge fields with the same value of the index. The diagrams in fig. 9(b),(c) are diagrams which do not actually appear due to the planarity restriction, but are diagrams that need to be subtracted explicitly. In other words, it is convenient to add and subtract the diagrams in fig. 9(b),(c). These diagrams added to the rest of the planar diagrams give the exponentiation of the one loop result.

Diagrams fig. 9(a) and fig. 9(c) have UV divergencies that cancel. Diagrams fig. 9(b) and fig. 9(c) have IR divergencies which also cancel each other. The diagrams fig. 9(c) contains a single power of the distance and it is thus zero in dimensional regularization. Thus we only need to consider explicitly the diagrams (a) and (c). The one loop gluon propagator has the form

$$
\frac{g^{2}}{2} \frac{1}{p^{2}}\left(1-4 \lambda \frac{\Gamma(\epsilon) \Gamma(2-\epsilon) \Gamma(1-\epsilon)}{(4 \pi)^{2-\epsilon} \Gamma(3-\epsilon)} \frac{1}{p^{2 \epsilon}}\right)
$$

where we wend to $D=4-2 \epsilon$ dimensions. We will find it convenient to use the following formula

$$
I_{\alpha, D}=\int \frac{d^{D-1} p}{(2 \pi)^{D-1}} p^{-\alpha} e^{i \vec{p} \vec{y}}=y^{\alpha-D+1} \pi^{-\frac{D-1}{2}} 2^{-\alpha} \frac{\Gamma\left[\frac{D-\alpha-1}{2}\right]}{\Gamma\left[\frac{\alpha}{2}\right]}
$$

We then find that the one loop correction to the gluon propagator in (B.4) gives us a result of the form

$$
(a)=-T \frac{\lambda^{2}}{2} \frac{\Gamma(\epsilon) \Gamma(2-\epsilon) \Gamma(1-\epsilon)}{(4 \pi)^{2-\epsilon} \Gamma(3-2 \epsilon)} 4 I_{2+2 \epsilon, D}, \quad D=4-2 \epsilon
$$


were $T$ is the length of the dimension along the loop.

The structure of the (b) diagram is

$$
(b)=T \int_{0}^{\infty} d t_{2} \int_{-\infty}^{\infty} d t_{3} \int_{t_{3}}^{\infty} d t_{4} G\left(t_{4}^{2}+y^{2}\right) G\left(\left(t_{2}-t_{3}\right)^{2}+y^{2}\right)
$$

where we have set $t_{1}=0$ using translation invariance. We also are using the expression for the propagator in position space $G$. We can go to Fourier space by using the following expression for the step function $\Theta(t)$

$$
\Theta(t)=\frac{1}{i} \int_{-\infty}^{\infty} \frac{d k}{2 \pi} \frac{1}{k-i \eta} e^{i k t}
$$

where $\eta$ is small. We can then use this integral to enforce the ordering we have in the fig. 8(b). We find (even though we use the index 0, we are in Euclidean space)

$$
\begin{aligned}
(b) & =-\frac{\lambda^{2}}{4} T \frac{1}{i^{2}} \int d t_{2} d t_{3} d t_{4} \int \frac{d k}{2 \pi} \frac{d k^{\prime}}{2 \pi} \frac{d q_{0} d p_{0}}{(2 \pi)^{2}} \frac{d^{D-1} p}{(2 \pi)^{D-1}} \frac{d^{D-1} q}{(2 \pi)^{(D-1)}} \times \\
& \times e^{i q_{0} t_{4}} e^{i k t_{2}} e^{i p_{0}\left(t_{3}-t_{2}\right)} e^{i k^{\prime}\left(t_{4}-t_{3}\right)} e^{i(\vec{p}-\vec{q}) \vec{y}} \frac{1}{k-i \eta} \frac{1}{k^{\prime}-i \eta} \frac{1}{q_{0}^{2}+q^{2}} \frac{1}{p_{0}^{2}+p^{2}}
\end{aligned}
$$

The minus sign arises from the fact that we are subtracting this contribution. We can now do the integrals over $t_{2}, t_{3}, t_{4}$ which give $(2 \pi)^{3} \delta\left(k-p_{0}\right) \delta\left(p_{0}-k^{\prime}\right) \delta\left(q_{0}+k^{\prime}\right)$. We now then need to the integral over $p_{0}$ which is of the form

$$
\int \frac{d p_{0}}{2 \pi} \frac{1}{\left(p_{0}-i \eta\right)^{2}\left(p_{0}^{2}+p^{2}\right)\left(p_{0}^{2}+q^{2}\right)}=-\frac{1}{2 q^{3} p^{2}}-\frac{1}{2 q^{2} p^{3}}+\frac{1}{2 p^{2} q^{2}(p+q)}
$$

Thus the end result for the (b) type diagrams seems to be

$$
\begin{aligned}
(b)= & \frac{\lambda^{2} T}{4}\left(-I_{2, D} I_{3, D}+\tilde{I}+o(\epsilon)\right), \\
& \tilde{I}=\frac{1}{2} \int \frac{d^{3} p}{(2 \pi)^{3}} \int \frac{d^{3} q}{(2 \pi)^{3}} \frac{e^{i(\vec{p}+\vec{q}) \cdot \vec{y}}}{q^{2} p^{2}(p+q)}=\frac{1}{y} \frac{\log 2}{2^{3} \pi^{3}}
\end{aligned}
$$

where we have set $D=4$ in the last term since it is not divergent. Summing the contributions of (a) and (b) and taking $\epsilon \rightarrow 0$ we get

$$
\log \langle W\rangle=\frac{1}{8 \pi}\left(\lambda-\frac{\lambda^{2}}{4 \pi^{2}}+\cdots\right) \frac{T}{y}
$$

where the dots indicates terms of order $\lambda^{3}$ (or possibly terms of order $\lambda^{3} \log \lambda$ as was found in QCD [27]). 


\section{References}

[1] L. F. Alday and J. Maldacena, "Gluon scattering amplitudes at strong coupling," JHEP 0706, 064 (2007) [arXiv:0705.0303 [hep-th]].

[2] S. Abel, S. Forste and V. V. Khoze, arXiv:0705.2113 [hep-th]. E. I. Buchbinder, arXiv:0706.2015 [hep-th]. M. Kruczenski, R. Roiban, A. Tirziu and A. A. Tseytlin, arXiv:0707.4254 [hep-th]. A. Jevicki, C. Kalousios, M. Spradlin and A. Volovich, arXiv:0708.0818 [hep-th]. Z. Komargodski and S. S. Razamat, "Planar quark scattering at strong coupling and universality," arXiv:0707.4367 [hep-th]. A. Mironov, A. Morozov and T. N. Tomaras, arXiv:0708.1625 [hep-th]. H. Kawai and T. Suyama, arXiv:0708.2463 [hep-th]. S. G. Naculich and H. J. Schnitzer, arXiv:0708.3069 [hep-th].

[3] J. McGreevy and A. Sever, "Quark scattering amplitudes at strong coupling," arXiv:0710.0393 [hep-th].

[4] Z. Bern, L. J. Dixon and V. A. Smirnov, "Iteration of planar amplitudes in maximally supersymmetric Yang-Mills theory at three loops and beyond," Phys. Rev. D 72, 085001 (2005) arXiv:hep-th/0505205.

[5] J. M. Drummond, J. Henn, G. P. Korchemsky and E. Sokatchev, "On planar gluon amplitudes/Wilson loops duality," arXiv:0709.2368 [hep-th].

[6] need to supply reference firstconformal.

[7] J. M. Drummond, G. P. Korchemsky and E. Sokatchev, "Conformal properties of four-gluon planar amplitudes and Wilson loops," arXiv:0707.0243 [hep-th].

[8] D. Nguyen, M. B. Spradlin and A. Volovich, "New Dual Conformally Invariant OffShell Integrals," arXiv:0709.4665 [hep-th].

[9] Z. Bern, M. Czakon, L. J. Dixon, D. A. Kosower and V. A. Smirnov, "The Four-Loop Planar Amplitude and Cusp Anomalous Dimension in Maximally Supersymmetric Yang-Mills Theory," Phys. Rev. D 75, 085010 (2007) arXiv:hep-th/0610248. Z. Bern, J. J. M. Carrasco, H. Johansson and D. A. Kosower, "Maximally supersymmetric planar Yang-Mills amplitudes at five loops," arXiv:0705.1864 [hep-th].

[10] J. M. Maldacena, "Wilson loops in large N field theories," Phys. Rev. Lett. 80, 4859 (1998) arXiv:hep-th/9803002.

[11] S. J. Rey and J. T. Yee, "Macroscopic strings as heavy quarks in large $\mathrm{N}$ gauge theory and anti-de Sitter supergravity," Eur. Phys. J. C 22, 379 (2001) arXiv:hepth/9803001].

[12] A. Brandhuber, P. Heslop and G. Travaglini, "MHV Amplitudes in N=4 Super YangMills and Wilson Loops," arXiv:0707.1153 [hep-th].

[13] M. T. Grisaru and H. N. Pendleton, "Some Properties Of Scattering Amplitudes In Supersymmetric Theories," Nucl. Phys. B 124, 81 (1977). S. J. Parke and T. R. Taylor, "Perturbative QCD Utilizing Extended Supersymmetry," Phys. Lett. B 157, 81 (1985) [Erratum-ibid. 174B, 465 (1986)]. 
[14] M. Kruczenski, "A note on twist two operators in N $=4 \mathrm{SYM}$ and Wilson loops in Minkowski signature," JHEP 0212, 024 (2002) arXiv:hep-th/0210115.

[15] O. Aharony, A. Fayyazuddin and J. M. Maldacena, "The large N limit of $\mathrm{N}=2,1$ field theories from three-branes in F-theory," JHEP 9807, 013 (1998) arXiv:hepth/9806159.

[16] A. Karch and E. Katz, "Adding flavor to AdS/CFT," JHEP 0206, 043 (2002) arXiv:hep-th/0205236.

[17] N. Beisert, B. Eden and M. Staudacher, "Transcendentality and crossing," J. Stat. Mech. 0701 (2007) P021 arXiv:hep-th/0610251.

[18] A. V. Kotikov, L. N. Lipatov, A. I. Onishchenko and V. N. Velizhanin, "Three-loop universal anomalous dimension of the Wilson operators in $\mathrm{N}=4$ SUSY Yang-Mills model," Phys. Lett. B 595, 521 (2004) [Erratum-ibid. B 632, 754 (2006)] arXiv:hepth/0404092].

[19] S. S. Gubser, I. R. Klebanov and A. M. Polyakov, "A semi-classical limit of the gauge/string correspondence," Nucl. Phys. B 636, 99 (2002) arXiv:hep-th/0204051.

[20] Z. Bern, M. Czakon, L. J. Dixon, D. A. Kosower and V. A. Smirnov, Phys. Rev. D 75, 085010 (2007) arXiv:hep-th/0610248]. S. Frolov and A. A. Tseytlin, JHEP 0206, 007 (2002) arXiv:hep-th/0204226]. R. Roiban and A. A. Tseytlin, arXiv:0709.0681 [hep-th]. M. K. Benna, S. Benvenuti, I. R. Klebanov and A. Scardicchio, Phys. Rev. Lett. 98, 131603 (2007) arXiv:hep-th/0611135]. B. Basso, G. P. Korchemsky and J. Kotanski, arXiv:0708.3933 [hep-th].

[21] J. K. Erickson, G. W. Semenoff and K. Zarembo, "Wilson loops in N = 4 supersymmetric Yang-Mills theory," Nucl. Phys. B 582, 155 (2000) arXiv:hep-th/0003055.

[22] A. Pineda, "The static potential in $\mathrm{N}=4$ supersymmetric Yang-Mills at weak coupling," arXiv:0709.2876 [hep-th].

[23] N. Drukker, D. J. Gross and H. Ooguri, "Wilson loops and minimal surfaces," Phys. Rev. D 60, 125006 (1999) arXiv:hep-th/9904191.

[24] N. Drukker and S. Kawamoto, "Small deformations of supersymmetric Wilson loops and open spin-chains," JHEP 0607, 024 (2006) arXiv:hep-th/0604124.

[25] S. S. Gubser, I. R. Klebanov and A. M. Polyakov, "Gauge theory correlators from non-critical string theory," Phys. Lett. B 428, 105 (1998) arXiv:hep-th/9802109.

[26] N. Drukker, D. J. Gross and A. A. Tseytlin, "Green-Schwarz string in AdS(5) x S(5): Semiclassical partition function," JHEP 0004, 021 (2000) arXiv:hep-th/0001204.

[27] T. Appelquist, M. Dine and I. J. Muzinich, "The Static Limit Of Quantum Chromodynamics," Phys. Rev. D 17, 2074 (1978). 